\title{
A benchmark for validation of numerical results in sheet metal forming
}

\author{
Abel D. Santos ${ }^{\mathrm{a}, *}$, Ana Reis ${ }^{\mathrm{a}}$, J.F. Duarte ${ }^{\mathrm{a}}$, Pedro Teixeira ${ }^{\mathrm{b}}$, A. Barata Rocha ${ }^{\mathrm{a}}$, \\ M.C. Oliveira ${ }^{\mathrm{c}}$, J.L. Alves $^{\mathrm{c}}$, Luis Menezes ${ }^{\mathrm{c}}$ \\ ${ }^{a}$ FEUP-DEMEGI, Department of Mechanical Engineering and Industrial Management, Porto University, Porto 4200465 , Portugal \\ ${ }^{\mathrm{b}}$ INEGI, Institute of Mechanical Engineering and Industrial Management, Portugal \\ ${ }^{\mathrm{c}}$ FCTUC, Department of Mechanical Engineering, University of Coimbra, Portugal
}

\begin{abstract}
Advances in FE codes for simulation of sheet metal forming processes have enabled its use during pre-production stage of a component. Some of recent developments include improvements and implementation of new material models of mechanical behaviour. However, these developments and corresponding results need to be validated, which means being compared with experimental results. On the other hand, in order that experimental results are a reference data, they need to be tested to repeatability and need to be obtained with such characteristic preferably by different institutions.

In this paper a benchmark is used to present experimental results obtained by different institutions as well as simulation results of the same benchmark. Different materials are used, namely an aluminium alloy (Al6016-T4) and two steels (a mild steel-DC06-and a high strength steel-DP600).
\end{abstract}

(C) 2004 Elsevier B.V. All rights reserved.

Keywords: Sheet metal forming; Finite element modelling; Reference experimental results

\section{Introduction}

The presented work is part of an international project3DS, Digital Die Design System [1]. One of the goals of this project is to improve and test the ability of numerical modelling codes to predict sheet metal forming defects as well as the final stamped geometry after springback.

In order to improve such prediction this project has planned that numerical codes will use new material models and better representation of friction properties. To test and validate these models and corresponding results a strong experimental component has been defined and a set of benchmarks has been developed. To avoid scatter in experimental results a special care has been taken to set a series of standard procedures in experimental tests [2].

It has been also included in this project the development of software, which is able to perform the comparison of geometries originated from CAD, measurements and results from numerical modelling. This task has been developed by the Japanese partners [3] of this project and such software will be used in this paper for comparison of results.

\footnotetext{
* Corresponding author. Fax: +351-22-5081445.

E-mail addresses: abel@fe.up.pt (A.D. Santos), cetecop@inegi.up.pt (P. Teixeira), luis.menezes@mail.dem.uc.pt (L. Menezes).
}

\section{Experimental conditions}

In order to obtain a representative set of reference experimental results it has been defined that different working group would perform several identical tests with distinct but identical tools in different institutions. Also to assure the uniformity and constancy of experimental variables it has been defined the methodology to perform the experiments and the quantification of experimental values. They include cutting of blanks from sheets, rolling direction, lubricant, lubrication method and quantity, blank holder force and ram speed, labelling and storage of parts, etc.

In this paper the selected benchmark, for which we present results, is what we will call "rail 2". Rail 2, as seen in Fig. 2, is a geometry that is prone to develop "3D springback", change in shape along the cross direction and curvature along the longitudinal direction. This benchmark has been set with an initial blank holder force of $90 \mathrm{kN}$ and the punch stroke is $60 \mathrm{~mm}$. The section profile in the middle part of rail is the result to be compared.

\subsection{Blanks}

The blanks are obtained from rolled sheets with known mechanical properties. Blank dimensions are $300 \mathrm{~mm} \times 300 \mathrm{~mm}$ with an accuracy of $0.5 \mathrm{~mm}$. Another 


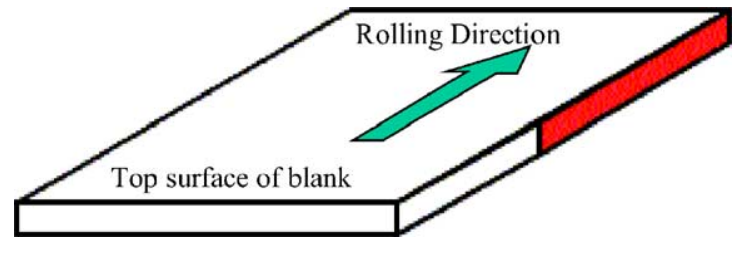

Fig. 1. Marking of blank.

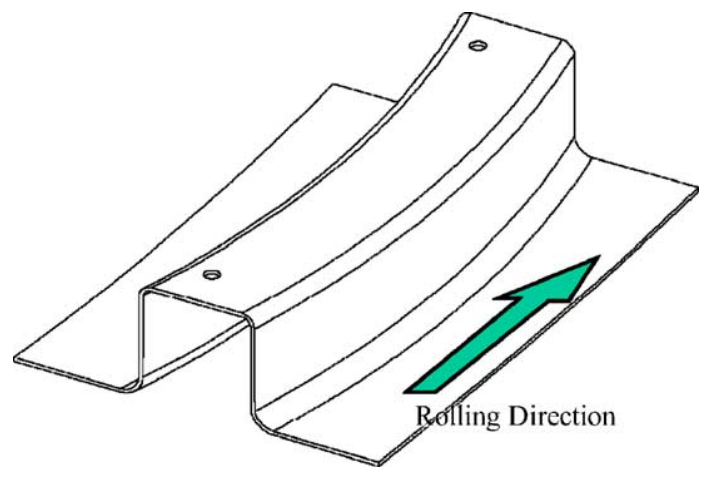

Fig. 2. Rolling direction.

aspect to consider is the blank edge effect caused by the rolling process. To eliminate this effect in blanks, material located within $50 \mathrm{~mm}$ from the edge is not used in these tests. All benchmarks are correctly marked (Fig. 1), to easily identify the sheet upper face as well as its rolling direction. This sheet rolling direction corresponds to longitudinal direction of rail as seen in Fig. 2.

\subsection{Lubrication}

In experimental testing it is very important to ensure uniformity and consistency of lubrication. The lubricant amounts were defined for each material, according to Table 1. As seen, there are two levels of lubricant amount, smaller for steels, bigger for aluminium.

In order to try to guarantee a correct amount of lubricant and its uniform distribution in blank, a method for lubrication has been followed by using a syringe to store and put an initial quantity of lubricant on blank, as well as a sponge to spread such lubricant. The steps for this method are the following:

1. Measure the sponge and syringe with oil (Fig. 3);

2. Put oil on sheet with syringe (Fig. 4);

Table 1

Lubricant conditions

\begin{tabular}{lllll}
\hline \multicolumn{2}{l}{ Material } & \multirow{2}{l}{ Oil } & \multicolumn{2}{l}{ Quantity } \\
\cline { 1 - 1 } Code & Name & & $\mathrm{g} / \mathrm{m}^{2} / \mathrm{side}$ & $\mathrm{g} /$ side $(0.3 \mathrm{~m} \times 0.3 \mathrm{~m})$ \\
\hline $\mathrm{M}$ & DC06 & Quaker 6130 & 1.5 & 0.135 \\
$\mathrm{H}$ & ZSTE340 & Fuchs 4107S & 1.5 & 0.135 \\
$\mathrm{D}$ & DP600 & Quaker 6130 & 1.5 & 0.135 \\
5 & $5182-\mathrm{O}$ & Fina 5754 & 2.5 & 0.225 \\
6 & 6016-T4 & Fina 5754 & 2.5 & 0.225 \\
\hline
\end{tabular}

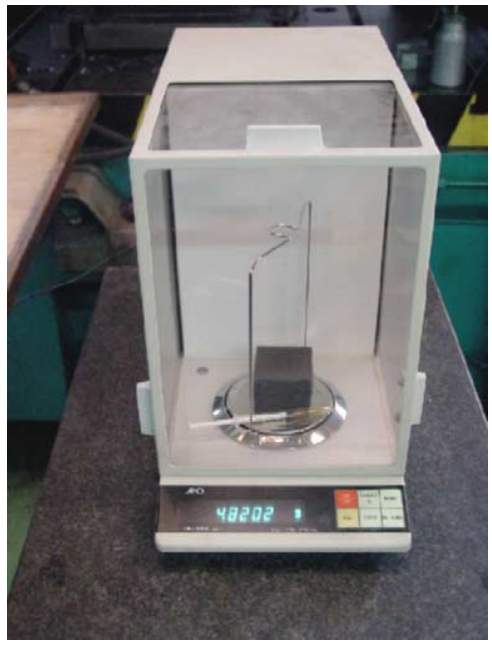

Fig. 3. Weighting sponge and syringe.

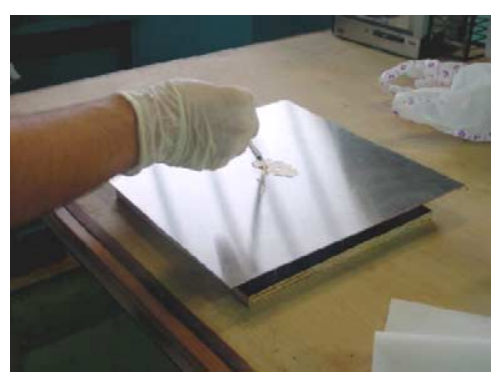

Fig. 4. Oiling blank with syringe.

3. Spread oil on sheet surface by using a sponge (Fig. 5);

4. Measure again the sponge and syringe (Fig. 6);

5. Iteration between steps 3 and 4 until correct amount of oil is left on blank.

\subsection{Bank holder force}

The experimental tests have been defined with three different blank holder forces: 90, 200 and $300 \mathrm{kN}$. This B.H. force is assured by six nitrogen gas springs, connected between them, thus giving a better distribution of force under B.H. plate. Also the gas pressure assures a smaller increase of B.H. force during stamping, when compared with usual steel springs.

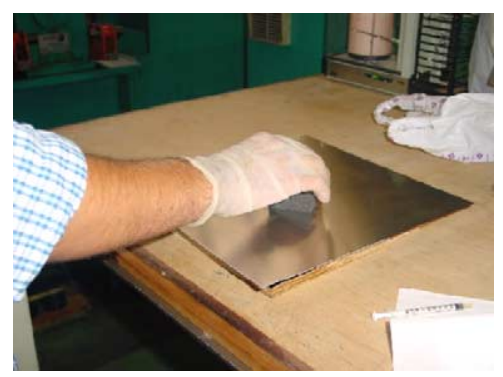

Fig. 5. Spreading oil with sponge. 


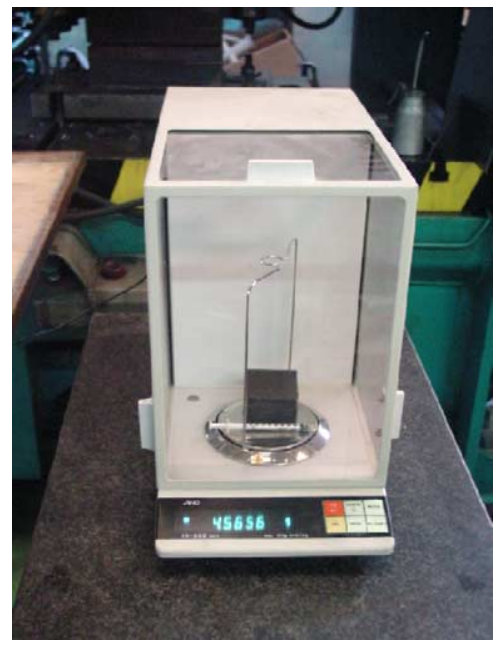

Fig. 6. Weighting sponge and syringe.

\subsection{Measurement of parts}

The comparison of results among different groups is based mainly in section profiles of final parts, as well as force/stroke diagrams, both experimental and numerical. In order that a section from different groups may be easily superposed for comparison is important a unique definition of the coordinate system to be used, and for experiments a standard support for parts location during measurement. For this benchmark (rail 2) four section profiles were defined (Fig. 7). During stamping, two holes are punched in top surface of parts. They are used to position parts in a jig for measurement (Fig. 8), and also they provide a direction for reference coordinate system. Fig. 9 shows the 3-point location for position of parts in the jig. As for the coordinate system, its definition is the following:

- reference origin, point $(0,0,0)$ is located in the front hole according to Fig. 10;

- $x$ direction is along longitudinal direction of rail, connecting the two holes centres;

- $z$-support pins create the location for $x y$ plane (Fig. 9).

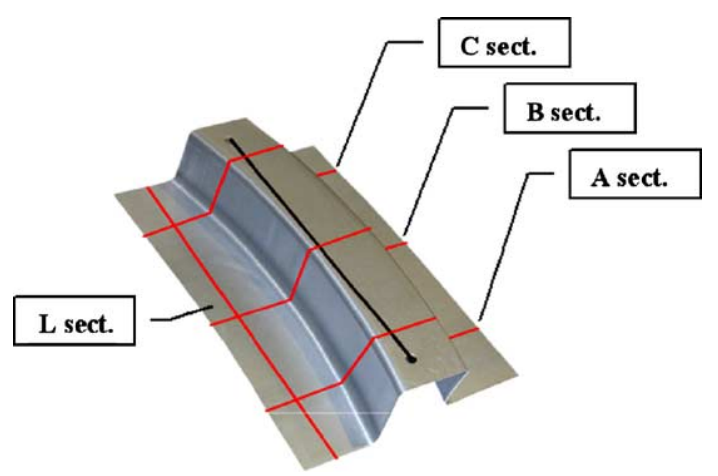

Fig. 7. Profiles to be evaluated.

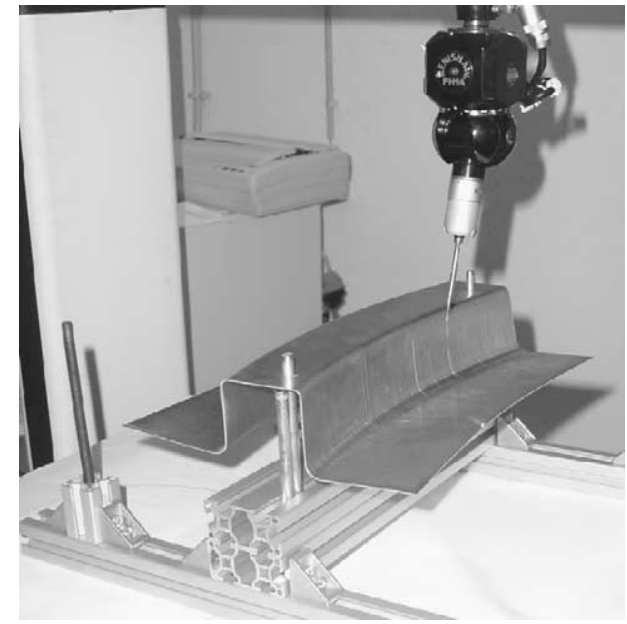

Fig. 8. Profile measurement.

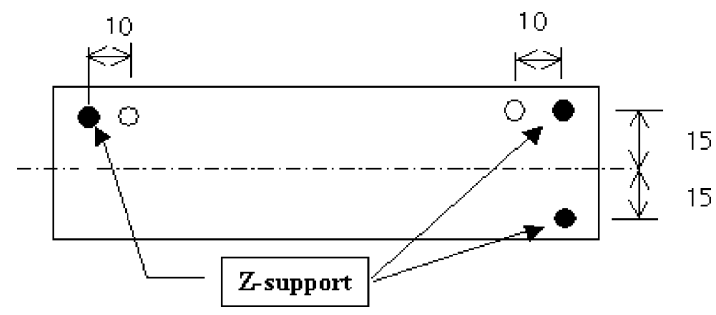

Fig. 9. Supporting pins.

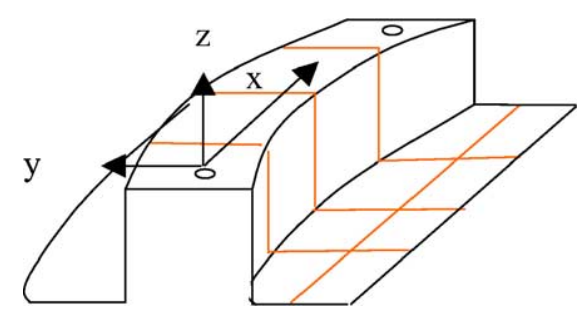

Fig. 10. Reference system.

\section{The finite element code-DD3IMP}

DD3IMP is the name of the finite element code developed at CEMUC (Centre at FCTUC) to simulate sheet metal forming processes. The code DD3IMP uses a mechanical model that takes into account the large elastoplastic strains and rotations. The plastic behaviour of the material is described by the Hill's yield criterion with isotropic and kinematic work hardening, and by an associated flow rule. Several work hardening constitutive models have been implemented in order to allow a better description of the different material mechanical behaviour [4]. The Coulomb classical law models the frictional contact problem, which is treated with an augmented Lagrangian approach. A fully implicit algorithm of Newton-Raphson type is used to solve the non-linearities related with the frictional contact problems and the elastoplastic behaviour of the deformable body. The code uses solid finite elements. This represents 
Table 2

Global algorithm of DD3IMP code

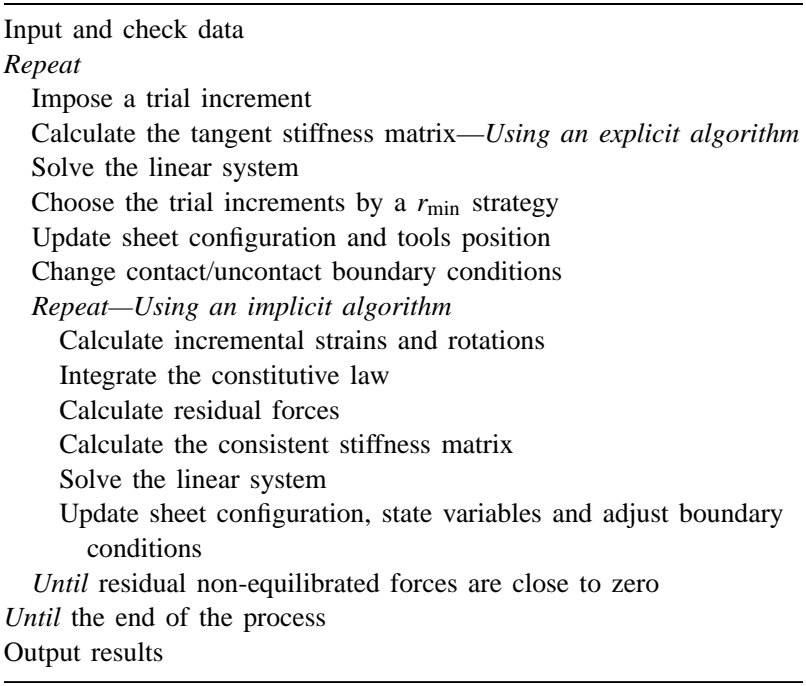

an enormous cost in terms of CPU time, but they allow an accurate calculation of the stress gradients through thickness of the sheet as well as the thickness evolution.

The global algorithm of this code is summarised in Table 2 .

The evolution of the deformation process is described by an updated Lagrangian formulation. In each load step, first solution for the incremental displacements, stresses and frictional contact forces is calculated with an explicit approach. An $r_{\text {min }}$ strategy is employed to impose limitations on the size of the time increments. Nevertheless, this first solution satisfies neither the variational principle nor the coherence condition. In order to guarantee that the equilibrium of the deformable body is satisfied this trial configuration is successively corrected using an implicit method. The configuration of the blank sheet and tools as well as all state variables are only updated when the structural balance is satisfied. The main characteristic of the global algorithm of DD3IMP code is the use of a single iterative loop to solve both non-linearities related with the mechanical behaviour and the contact with friction problem.

\subsection{Numerical modelling}

As already said, the selected deep drawing problem is the rail presented in Fig. 2. The tool and blank set-up is schematic represented in Fig. 11. The blank sheet is a square of $300.0 \mathrm{~mm}$ length and $1.0 \mathrm{~mm}$ thickness. Due to the symmetry only a half of the problem was considered in simulation. The finite element mesh is composed of 8-node hexahedrons combined with a selective reduced integration method. The average element size on the flat contact zone between the blank holder and the die has $6.0 \mathrm{~mm}$ and it is reduced to $3.0 \mathrm{~mm}$ in the other contact areas. Only one layer of elements is used in thickness. In DD3IMP the geometry of the forming tools is modelled by parametric Bézier sur-

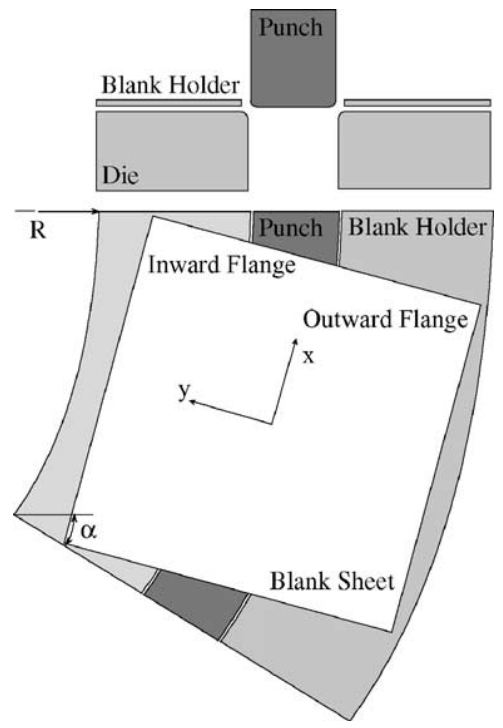

Fig. 11. Stamping tools geometry.

faces. The followed numerical schemes rely on a frictional contact algorithm that operates directly on the parametric Bézier surfaces.

In this work the material is assumed to have an isotropic and kinematic work hardening. For the steels the isotropic work hardening is described by the Swift law, Eq. (1), and for the aluminium alloy by the Voce law, Eq. (2):

$Y=C\left(\varepsilon_{0}+\bar{\varepsilon}^{p}\right)^{n}$

$Y=Y_{0}+R_{\text {sat }}\left[1-\exp \left(-C_{R} \bar{\varepsilon}^{p}\right)\right]$

where $C, \varepsilon_{0}$ and $n$ for the Swift law, as well as $Y_{0}, R_{\text {sat }}$ and $C_{R}$ for the Voce law are the material parameters determined from standard mechanical tests. $Y$ is the flow stress while $\bar{\varepsilon} p$ is the equivalent plastic strain given by:

$\bar{\varepsilon}^{p}=\int_{0}^{1} \dot{\bar{\varepsilon}}^{p} \mathrm{~d} t$

where $\dot{\bar{\varepsilon}} p$ is the equivalent plastic strain rate. The Lemaître and Chaboche saturation law describes the kinematic hardening:

$\stackrel{\mathbf{X}}{=}=C_{X}\left[\frac{X_{\text {sat }}}{\bar{\sigma}}\left(\sigma^{\prime}-\mathbf{X}\right)-\mathbf{X}\right] \dot{\bar{\varepsilon}}^{p} \quad$ with $\mathbf{X}(0)=\mathbf{0}$

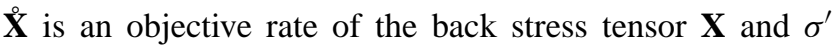
the deviatoric stress tensor. $X_{\text {sat }}$ characterises the saturation value of the kinematic hardening, while the parameter $C_{X}$ characterizes the rate of approaching the saturation. The equivalent stress definition assumes the form:

$\bar{\sigma}=\sqrt{\left(\sigma^{\prime}-\mathbf{X}\right): \mathbf{M}:\left(\sigma^{\prime}-\mathbf{X}\right)}$,

where $\mathbf{M}$ is a fourth-order symmetric anisotropy tensor associated to the Hill's 48-yield criterion, used to describe the orthotropic anisotropy of the rolled sheet. The mechanical 


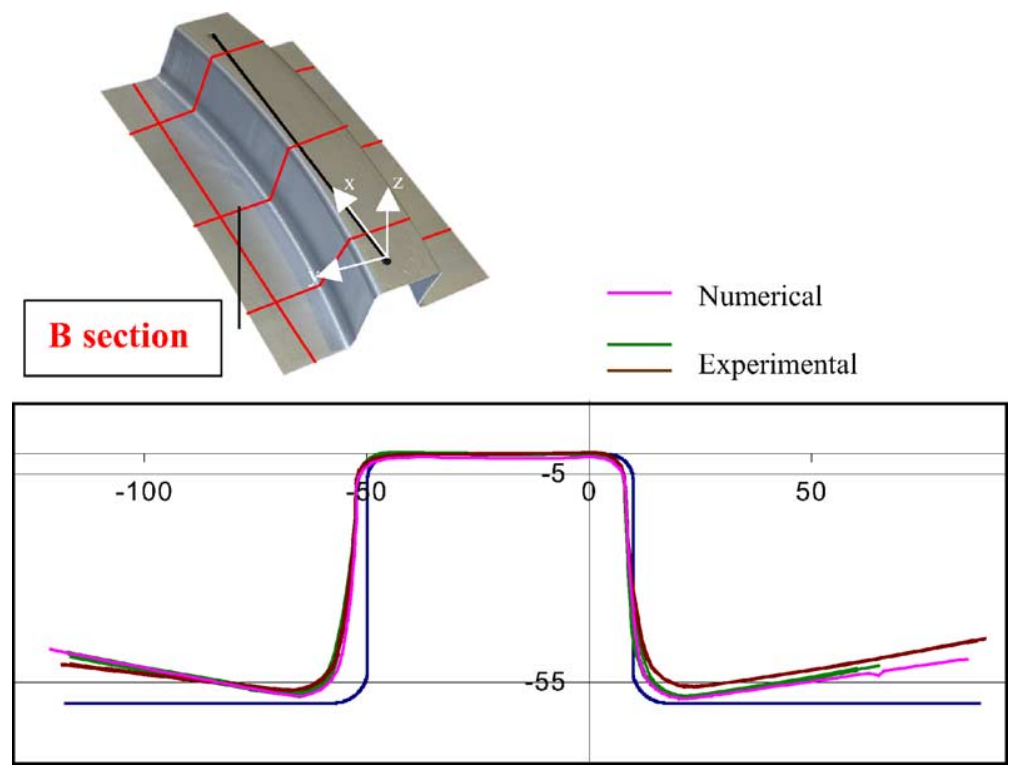

Fig. 12. Steel DP600, experimental and numerical profiles-B section.
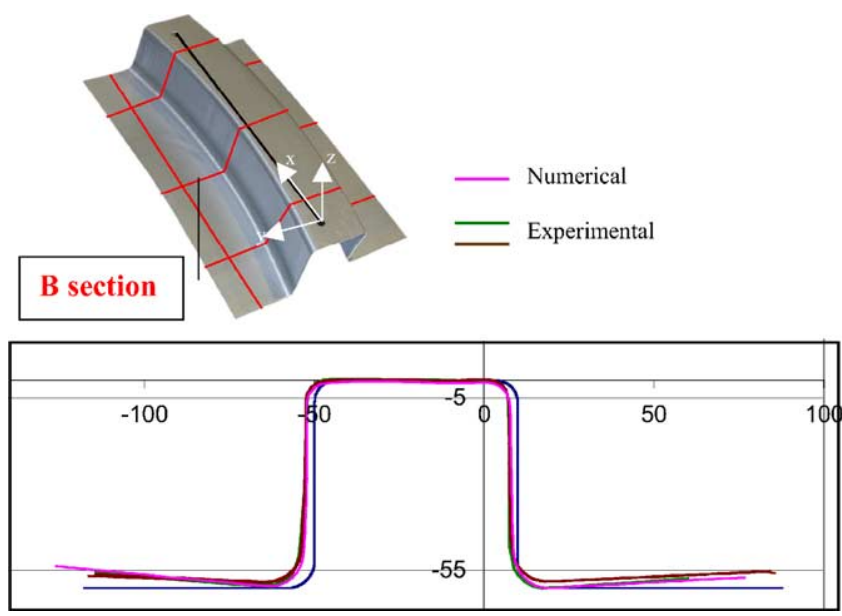

Fig. 13. Steel DC06, experimental and numerical profiles-B section.

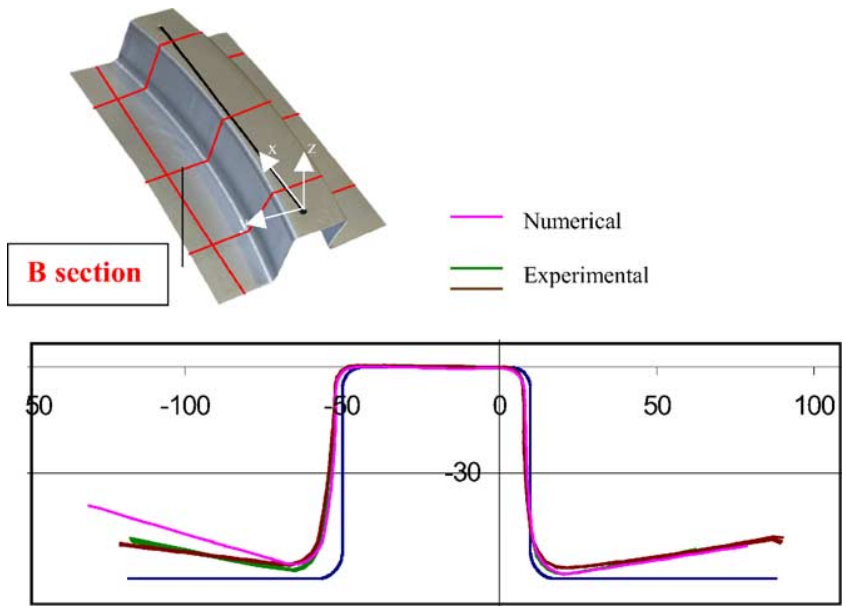

Fig. 14. Aluminium 6016, experimental and numerical profiles-B section. characterization as well as the tuning of the material parameters for the work hardening constitutive models evaluated in the present work was performed by LPMTM [5].

The tests were performed with a constant friction coefficient for all tools of 0.10 .

\section{Comparison between numerical and experimental results}

Comparisons are performed for one aluminium alloy (A16016) and two steels (a mild steel, DC06 and a high

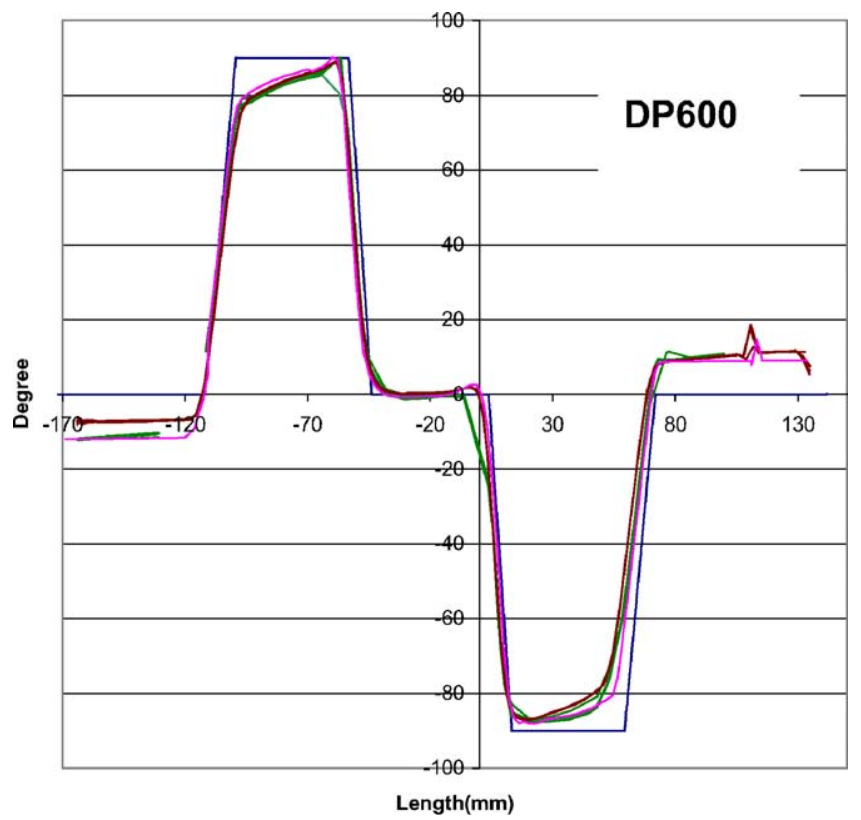

Fig. 15. Steel DP600, tangent angle variation of profiles-B section. 


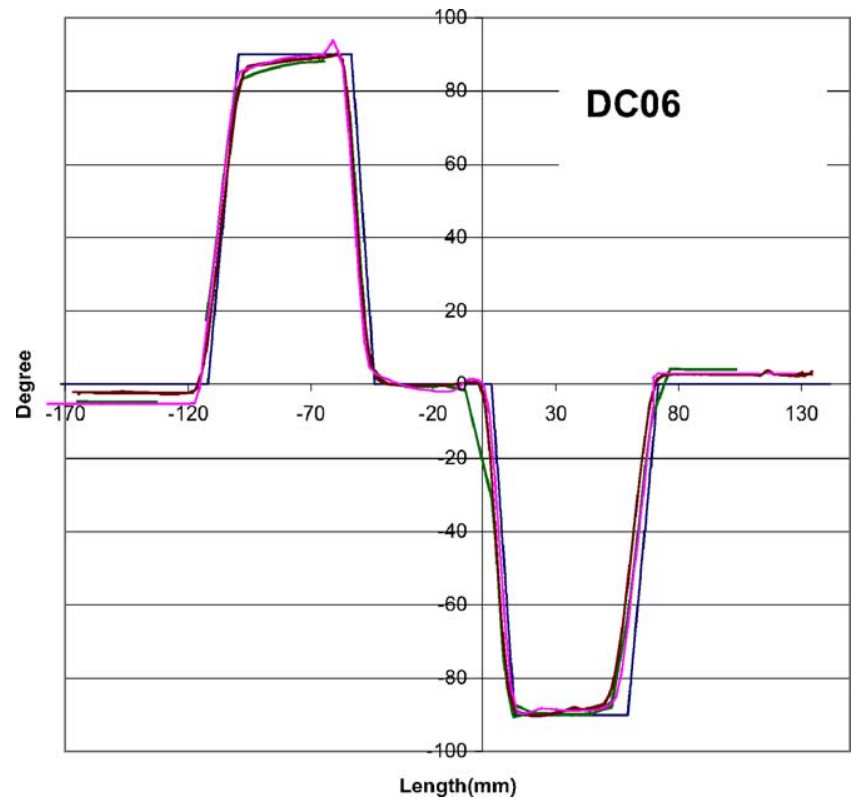

Fig. 16. Steel DC06, tangent angle variation of profiles-B section.

strength steel, DP600). Although four institutions have performed experimental tests, next results for aluminium show only those obtained by partners Pechiney and FEUP, since these are the most consistent to use for comparison with numerical results.

Figs. 12-14 show profiles obtained in stamped parts, both experimental and numerical, respectively for DP600, DC06 and Al6016.

Figs. 15-17 show the same results, but profiles are expressed as a function of angle variation along its length, which may give additional information regarding the differences of results.

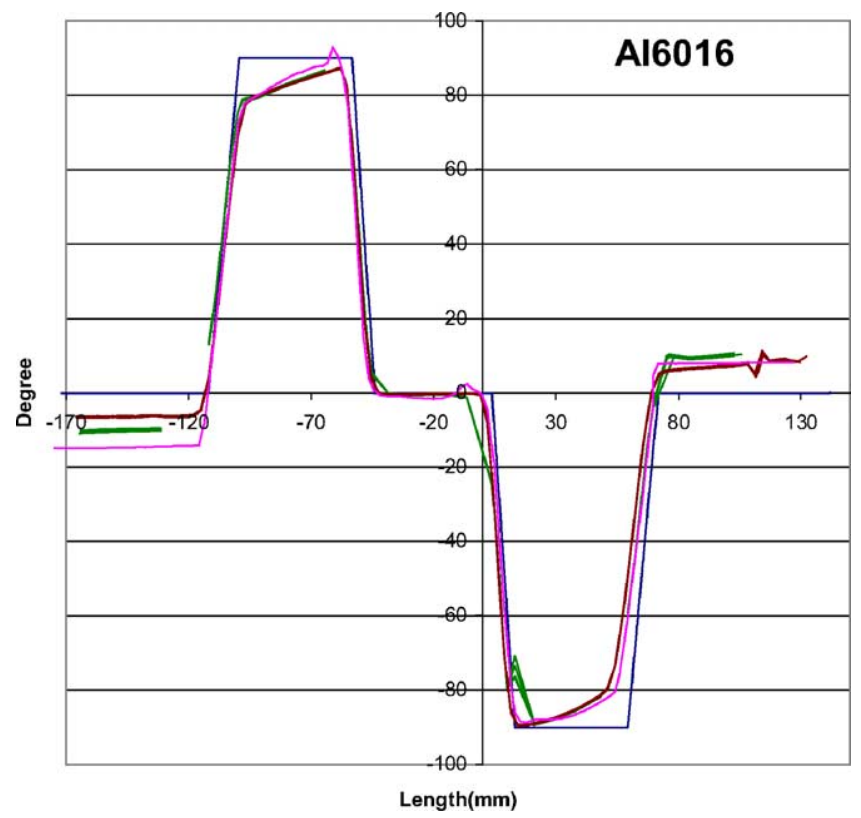

Fig. 17. Aluminium 6016, tangent angle variation of profiles-B section.
In this way we may understand if difference of profiles comes from punch radius (sheet bending), or vertical wall (bending/unbending) or die radius (bending).

From the three series of results (three materials), it may be seen that DC06 shows the best fitting for both experimental and experimental/numerical results.

Concerning the other materials there is some small dispersion in experimental results. For these materials, numerical results fit better with experiments on one side of the part than on the other. It seems also that flange length on numerical and experimental parts may differ, which suggests a closer investigation on this detail and its possible relation with observed differences.

\section{Conclusions}

A benchmark has been presented, which intend to be used to get experimental reference data for numerical validation. The scatter obtained in experimental results from different institutions suggests additional investigation to understand its reason and why is more evident for different materials.

The results presented herein show that numerical results may follow closely the experimental ones, which mean a good modelling of springback prediction for different materials.

\section{Acknowledgements}

The work herein presented was funded by European Community through Growth Programme (contract G1RD-CT-2000-00104) and by Portuguese Foundation for Science and Technology through Programme POCTI (contract EME/35945/99). The authors are grateful for this support.

\section{References}

[1] A. Col, "Presentation of the 3DS Research Project", NUMISHEET'02, Jeju Island, Korea, 2002.

[2] A.D. Santos, J.F. Duarte, A. Reis, A.B. da Rocha, L. Menezes, M. Oliveira, A. Col, T. Ono, Towards standard benchmarks and reference data for validation and improvement of numerical simulation in sheet metal forming, J. Mater. Process. Technol. 125/126 (2002) 798-805.

[3] M \& M Research, NXT Evaluat. processor, http://www.mresearch.co.jp

[4] L.F. Menezes, S. Thuiller, P.Y. Manach, S. Bouvier, Influence of the work-hardening models on the numerical simulation of a reverse deep-drawing process, in: A.S. Khan, Oscar Lopez-Pamies (Eds.), Proceedings of the Ninth International Symposium on Plasticity and its Current Applications (Plasticity'02), Plasticity, Damage and Fracture at Macro, Micro and Nanoscales, Neat Press, Maryland, USA, 2001, pp. 331-333.

[5] C. Teodosiu, S. Bouvier, Selection and Identification of Elastoplastic Models for Materials used in the Benchmarks, in: 18-months Progress Report, “3DS, Digital Die Design System”, 2001. 